\title{
Blood sulfur-amino acid concentration reflects an impairment of liver transsulfuration pathway in patients with acute abdominal inflammatory processes
}

\author{
Juan R. Viña ${ }^{1 *}$, Angel Giménez ${ }^{2}$, Adelina Corbacho ${ }^{1}$, Inmaculada R. Puertes ${ }^{1}$, Elisa Borrás ${ }^{1}$, \\ Concha García ${ }^{1}$ and Teresa Barber ${ }^{1}$ \\ ${ }^{1}$ Dpto Bioquímica y Biología Molecular, Facultades de Medicina y Farmacia, Universidad de Valencia, Spain \\ ${ }^{2}$ Hospital de la Ribera, Alzira, Valencia, Spain
}

(Received 1 March 2000 - Revised 4 August 2000 - Accepted 21 September 2000)

\begin{abstract}
Whole-blood free amino acids were measured in a control group made up of eight healthy women fasted for $12 \mathrm{~h}$ and also in eight patients with acute pancreatitis, five patients with acute cholecystitis and seven patients with acute appendicitis. Blood was withdrawn immediately on admission to hospital and again $3 \mathrm{~d}$ later following a controlled peripheral parenteral nutrition diet; this is with the exception of the appendicitis group. L-Cystathionine and L-methionine concentrations were significantly higher in pancreatitis and appendicitis patients when compared with controls. In the pancreatitis and cholecystitis patients, L-serine concentration was also significantly higher when compared with controls. The L-homocysteine concentration was significantly higher only in the appendicitis group when compared with the control group. LCystine concentration was unchanged in all the patients studied when compared with control subjects. The L-methionine:L-cystine ratio was significantly higher and the L-glutamine:Lcystine ratio was significantly lower in all the patients when compared with controls. The blood S-amino acid pattern reflects an impairment in liver transsulfuration pathway during acute abdominal processes. This work supports the idea that the L-methionine: L-cystine and Lglutamine:L-cystine ratios can be taken as good markers to evaluate the S-amino acid metabolism and suggests the importance of using $\mathrm{N}$-acetylcysteine as a required nutrient in these situations.
\end{abstract}

L-Methionine: L-Cystine: $\gamma$-Cystathionase

L-Methionine is an essential amino acid in mammals because its $\mathrm{C}$ skeleton cannot be synthesized. Its daily turnover is higher than the dietary intake, therefore the recycling of L-methionine from homocysteine is important (Nunn, 1987; Storch et al. 1988). L-Homocysteine is at a branch point because it can be remethylated to methionine by the vitamin $\mathrm{B}_{12}$-dependent methionine synthase (5methyltetrahydrofolate-homocysteine methyltransferase) or it can be condensed with L-serine to form L-cystathionine through the transsulfuration pathway due to the effect of cystathionine $\beta$-synthase (Fig. 1). This enzyme uses vitamin $\mathrm{B}_{6}$ as a cofactor and it is activated by $S$ adenosylmethionine. The transsulfuration sequence is completed by a reaction catalyzed by $\gamma$-cystathionase, which also uses vitamin $B_{6}$ as a coenzyme and is responsible for the cleavage of L-cystathionine to yield Lcysteine, $\alpha$-ketobutyrate and $\mathrm{NH}_{4}{ }^{+}$; therefore, it catalyses an essential step in the transsulfuration pathway which allows the synthesis of L-cysteine from L-methionine (Finkelstein, 1990).

Liver $\gamma$-cystathionase has been cloned (Erickson et al. 1990; Lu et al. 1992) and is under tight regulation. The enzyme is not fully expressed in human premature neonates (Sturman et al. 1970; Viña et al. 1995) In rats, we have shown that after $3 \mathrm{~d}$ stress induced by surgery the activity was significantly lower than in controls. In these rats, the rate of L-cysteine synthesis from L-methionine was significantly higher in isolated hepatocytes from controls than in hepatocytes from stressed rats. The half-life of blood L- $\left[2(n)-{ }^{3} \mathrm{H}\right]$ methionine was significantly higher in rats submitted to surgical stress than in controls, and the blood L-methionine:L-cystine ratio was significantly higher in stressed rats when compared with controls. All these facts support the hypothesis that L-cysteine might be 


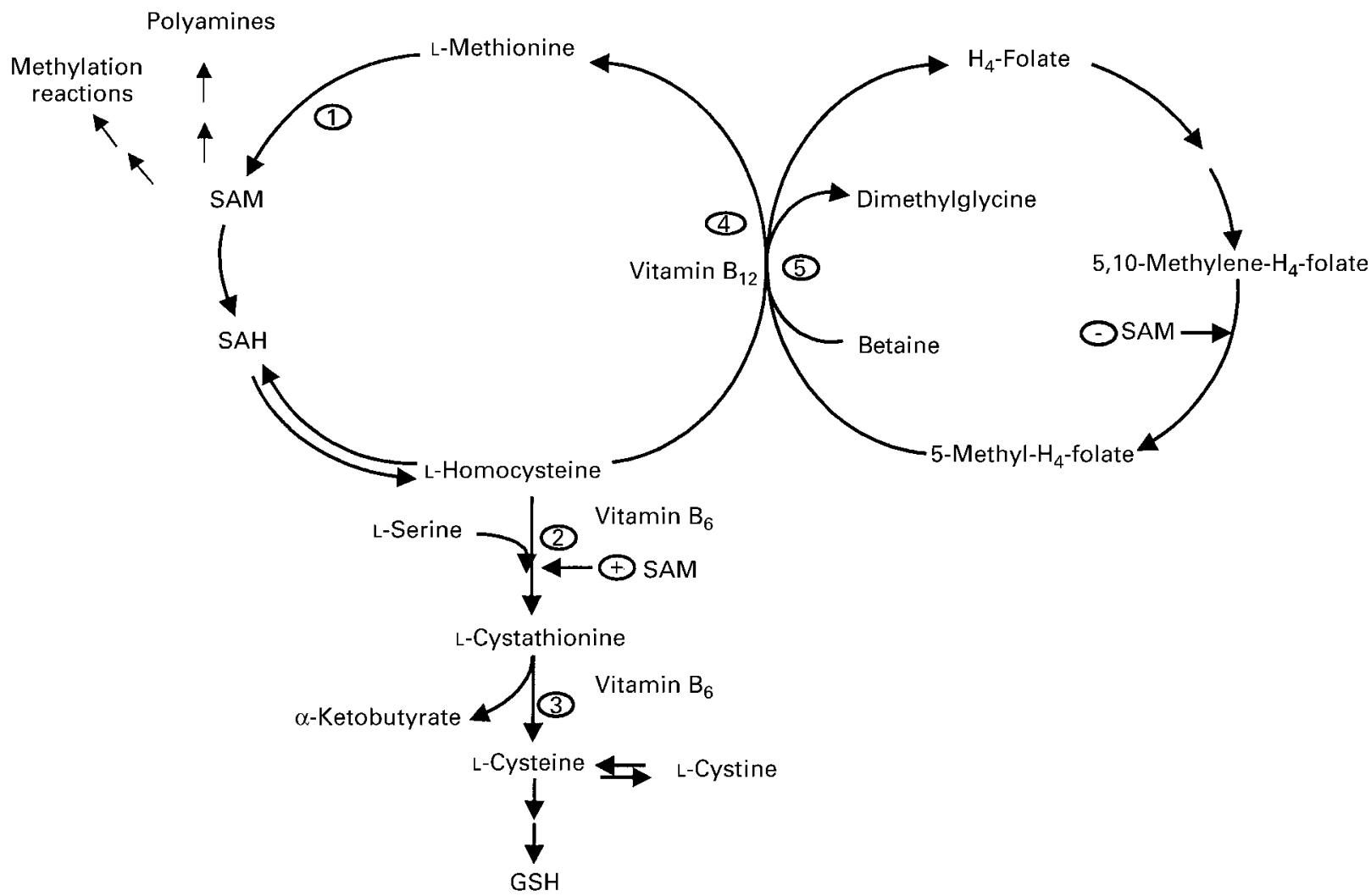

Fig. 1. Metabolic pathways describing L-methionine metabolism and the remethylation-transsulfuration of L-homocysteine. SAM, S-adenosylmethionine; $\mathrm{SAH}, S$-adenosylhomocysteine 1 , Methionine adenosyltransferase, 2 , cystathionine $\beta$-synthase (vitamin $\mathrm{B}_{6}$ dependent enzyme), 3 , $\gamma$-cystathionase (vitamin $B_{6}$ dependent enzyme), 4, methylfolate-homocysteine methyltransferase (methionine synthase) (vitamin $B_{12}$ dependent enzyme), 5 , betaine-homocysteine methyltransferase.

considered as an essential amino acid for man in cases of surgical stress (Viña et al. 1992).

Previous studies of S-amino acid metabolism during stress have been carried out on animals, so our aim was to determine if it was possible to detect an indication of the inhibition of liver $\gamma$-cystathionase in the whole blood of human subjects in stressful conditions, particularly as it is known that the activity in human liver is only one-fifth of that present in rat liver (Zlotkin \& Anderson 1982; Rao et al. 1990). It is interesting to point out that the activity of human liver cystathionine $\beta$-synthase, the other enzyme of the transsulfuration pathway, is also one-fifth of that present in rat liver (Kraus \& Rosenberg 1983; Asagi et al. 1996). In rats, $50 \%$ rat liver $\gamma$-cystathionase activity appears to be sufficient for transsulfuration, but $50 \%$ of the human adult level of hepatic $\gamma$-cystathionase may not be sufficient (Rao et al. 1990); therefore, a slight inhibition of the enzyme may change the blood S-amino acid pattern in man and could be a useful test which reflects the impairment of the transsulfuration pathway.

\section{Materials and methods}

\section{Subjects and study criteria}

Eight healthy female workers, with no history of metabolic disorders, employed at the Facultad de Medicina (University of Valencia, Spain) were selected at random to form the control group. Twenty patients from the emergency ward of the Hospital Lluís Alcanyís (Xátiva, Spain) were used for this study: eight patients with acute pancreatitis, five with acute cholecystitis and seven with acute appendicitis. On admission patients had a general screening which included a routine blood chemistry evaluation. Whole-blood free amino acid values were also evaluated. The first two groups received no food or water orally and they were under controlled peripheral parenteral nutrition for $3 \mathrm{~d}$ after their admission to hospital and then blood samples were obtained for blood free amino acid determination. The patients with acute pancreatitis had a nasogastric tube and intravenous analgesia, the cholecystitis patients had intravenous analgesia and broad spectrum antibiotic therapy; a nasogastric tube was used when vomiting was present. The patients with acute appendicitis received antibiotics before surgery was performed and afterwards blood was sampled for amino acid determination. All participants gave their consent and we obtained permission from the Ethical Committee of our Hospital.

\section{Peripheral parenteral nutrition}

The composition of the peripheral parenteral nutrition (per litre) was as follows: free amino acids $21.3 \mathrm{~g}$ (containing $(\mathrm{g} / \mathrm{l})$ : L-phenylalanine $1 \cdot 19$, L-serine $1 \cdot 06$, L-isoleucine 1.28, L-leucine 1.55 , L-lysine, $\mathrm{HCl} 1.54$, L-methionine 
Table 1. Clinical characteristics of patients on admission to hospital (Mean values with standard errors of the means)

\begin{tabular}{|c|c|c|c|c|c|c|c|c|}
\hline & \multicolumn{2}{|c|}{ Control ( $n 8)$} & \multicolumn{2}{|c|}{ Pancreatitis ( $n 8$ ) } & \multicolumn{2}{|c|}{ Cholecystitis ( $n 5$ ) } & \multicolumn{2}{|c|}{ Appendicitis ( $n 7$ ) } \\
\hline & Mean & SEM & Mean & SEM & Mean & SEM & Mean & SEM \\
\hline Age (years) & 32 & 5 & $66^{*}$ & 5 & $72^{*}$ & 2 & 31 & 6 \\
\hline BMI $\left(\mathrm{kg} / \mathrm{m}^{2}\right) \dagger$ & $23 \cdot 2$ & $1 \cdot 2$ & 25.9 & $1 \cdot 3$ & $30 \cdot 3^{*}$ & $2 \cdot 3$ & $24 \cdot 6$ & $1 \cdot 4$ \\
\hline WBC (cells $\left./ \mathrm{mm}^{3}\right)$ & 6707 & 502 & $16200^{*}$ & 1911 & $12620^{*}$ & 1184 & $11683^{*}$ & 448 \\
\hline Haemoglobin $(\mathrm{g} / \mathrm{l})$ & 128 & 5 & 145 & 6 & 143 & 14 & 138 & 3 \\
\hline Hematocrit index (\%) & $38 \cdot 0$ & $1 \cdot 3$ & $42 \cdot 1$ & $1 \cdot 8$ & 41.4 & $4 \cdot 1$ & $41 \cdot 8$ & 0.5 \\
\hline Lymphocytes (cells/mm³) & 2267 & 353 & 2101 & 389 & 1342 & 364 & 2615 & 512 \\
\hline Glucose (mg/l) & 898 & 33 & $1732^{*}$ & 203 & 1564 & 300 & 1010 & 55 \\
\hline Urea (mg/l) & 293 & 26 & 474 & 70 & 436 & 100 & 325 & 24 \\
\hline Creatinine (mg/l) & 9 & 0 & 11 & 1 & 14.5 & 3.0 & - & - \\
\hline Amylase ( $\mu \mathrm{kAT} / \mathrm{I})$ & $10 \cdot 5$ & $1 \cdot 3$ & $562 \cdot 9^{*}$ & $131 \cdot 2$ & - & - & - & - \\
\hline Albumin (g/l) & 44 & 0 & $41 \cdot 8$ & 1.5 & $35^{\star}$ & 1 & - & - \\
\hline AST $(\mu \mathrm{kAT} / \mathrm{l})$ & 2.5 & $0 \cdot 2$ & $31 \cdot 1$ & $15 \cdot 8$ & $26 \cdot 2$ & $18 \cdot 8$ & - & - \\
\hline ALT ( $(\mu \mathrm{kAT} / \mathrm{l})$ & 1.9 & 0.2 & $50 \cdot 1$ & $21 \cdot 2$ & $32 \cdot 8$ & $23 \cdot 2$ & - & - \\
\hline
\end{tabular}

WBC, white blood cell count; AST, aspartate aminotransferase; ALT, alanine aminotransferase.

Mean values were significantly different from those of the control group: ${ }^{\star} P<0.05$.

† BMI $\left(\mathrm{kg} / \mathrm{m}^{2}\right)$, normal 18.5-24.9, overweight 25.0-29.9, obese 30.0-39.9.

$0 \cdot 85$, L-valine $1 \cdot 23$, L-histidine $1 \cdot 02$, L-threonine $0 \cdot 89, \mathrm{~L}$ tryptophan $0 \cdot 38$, L-alanine $4 \cdot 40$, glycine $2 \cdot 19$, L-arginine 2.45, L-proline 1.45 and L-tyrosine 0.085 ), glucose $75 \mathrm{~g}$, $\mathrm{Na}^{+} 34.6 \mathrm{mmol}, \mathrm{K}^{+} 25 \mathrm{mmol}, \mathrm{Mg}^{2+} 1.25 \mathrm{mmol}$, chloride $44.6 \mathrm{mmol}$, phosphate $7.5 \mathrm{mmol}$. The non-protein energy: $\mathrm{N}(\mathrm{g})$ ratio was 358 , and $\mathrm{K}(\mathrm{mmol}): \mathrm{N}(\mathrm{g})$ ratio 7 .

\section{Amino acid determination}

Whole blood was collected in a heparinized syringe, and protein was precipitated by mixing $1 \mathrm{ml}$ blood and $1 \mathrm{ml}$ sulfosalicylic acid $(50 \mathrm{~g} / \mathrm{l})$ in $0.3 \mathrm{M}$-lithium citrate buffer $(\mathrm{pH}$ 2.8). Therefore, L-cysteine and L-cystine were measured together. The supernatant fraction $(50 \mu \mathrm{l})$ was injected into an LKB 4151 Alpha Plus Amino Acid Analyser (Amersham Pharmacia Biotech UK Ltd, Little Chalfont, Bucks., UK) for determination of amino acids. Blood was withdrawn from control subjects after overnight fasting, and in patients under controlled diet conditions the sampling was performed $4 \mathrm{~h}$ after the peripheral parenteral nutrition was stopped.

\section{Statistics}

One way ANOVA was performed. The homogeneity of the variances was analysed by the Levene test; in those cases in which the variances were unequal, the data were adequately transformed before ANOVA. The null hypothesis was accepted for all the values of these sets in which $F$ was nonsignificant at $P>0.05$. Second, the sets of data in which $F$ was significant were examined by the Bonferroni test at $P<0.05$. In Table 2 the statistical analysis was performed in three groups: the control $v$. the pancreatitis before and after nutrition, the control $v$. cholecystitis before and after nutrition, and control $v$. appendicitis. In the appendicitis group modified $t$ statistics were performed directly. Values in the text are means with standard errors of the means.

\section{Results}

\section{Feeding and general characteristics of subjects}

The biological characteristics of control patients and patients with inflammatory abdominal processes are shown in Table 1. The patients with acute pancreatitis had significantly higher levels of amylase, glucose and leucocytes when compared with control values. The patients with acute cholecystitis had significantly high leucocytes count and BMI, and low levels of albumin when compared with controls. The patients with acute appendicitis had high levels of leucocytes. With the exception of the patients with acute appendicitis who underwent surgery within hours of their admittance to hospital, all the patients received peripheral parenteral nutrition for $3 \mathrm{~d}$ with no food taken orally. This was done in order to have all the patients monitored with the same amount of macro- and micronutrient intake.

\section{Whole-blood amino acid concentrations}

Table 2 shows the concentrations of L-methionine, Lhomocysteine, L-serine, L-cystathionine, L-cystine, L-glutamine and L-glutamate in blood from patients with acute inflammatory abdominal processes such as pancreatitis, cholecystitis and appendicitis. Measurements were made when patients were admitted to hospital and then $3 \mathrm{~d}$ later, with the exception of the patients with appendicitis who underwent surgery after their admission to hospital. When patients were admitted to hospital, blood L-cystathionine and L-serine concentration was significantly higher in all the patients with acute inflammatory abdominal processes when compared with controls, with the exception of Lserine in appendicitis patients and L-cystathionine in cholecystitis patients. Similar results were found after $3 \mathrm{~d}$ controlled parenteral nutrition. In appendicitis patients, the L-methionine and L-homocysteine concentration was significantly higher than in controls. The L-methionine concentration in pancreatitis patients was significantly higher $3 \mathrm{~d}$ after admission to hospital. In all the patients 
Table 2. Whole-blood free amino acid concentration $(\mu \mathrm{mol} / \mathrm{l})$ from control subjects and from patients with acute abdominal inflammatory processes

(Mean values with standard errors of the means)

\begin{tabular}{|c|c|c|c|c|c|c|c|c|c|c|c|c|}
\hline & \multicolumn{2}{|c|}{ Control (n 8) } & \multicolumn{4}{|c|}{ Pancreatitis ( $n$ 8) } & \multicolumn{4}{|c|}{ Cholecystitis ( $n 5$ ) } & \multirow{2}{*}{\multicolumn{2}{|c|}{$\begin{array}{c}\text { Appendicitis }(n 7) \\
\text { On admission } \\
\text { to hospital }\end{array}$}} \\
\hline & \multirow[b]{2}{*}{ Mean } & \multirow[b]{2}{*}{ SEM } & \multicolumn{2}{|c|}{$\begin{array}{l}\text { On admission } \\
\text { to hospital }\end{array}$} & \multicolumn{2}{|c|}{$\begin{array}{c}3 \mathrm{~d} \text { after } \\
\text { controlled diet } \dagger\end{array}$} & \multicolumn{2}{|c|}{$\begin{array}{l}\text { On admission } \\
\text { to hospital }\end{array}$} & \multicolumn{2}{|c|}{$\begin{array}{c}3 \mathrm{~d} \text { after } \\
\text { controlled diet } \dagger\end{array}$} & & \\
\hline & & & Mean & SEM & Mean & SEM & Mean & SEM & Mean & SEM & Mean & SEM \\
\hline L-Serine & 169 & 5 & $392^{*}$ & 35 & $455^{\star}$ & 58 & $305^{\star}$ & 27 & $345^{\star}$ & 36 & 293 & 75 \\
\hline L-Cystine & 44 & 3 & 41 & 6 & 33 & 4 & 38 & 5 & 36 & 9 & 56 & 12 \\
\hline L-Homocysteine & 3 & 2 & 21 & 10 & 17 & 9 & \multicolumn{2}{|c|}{ ND } & \multicolumn{2}{|c|}{ ND } & $60^{*}$ & 18 \\
\hline L-Methionine & 10 & 5 & 25 & 7 & $40^{*}$ & 11 & 9 & 5 & 31 & 8 & $60^{*}$ & 16 \\
\hline L-Cystathionine & 11 & 4 & $44^{*}$ & 11 & $39^{*}$ & 6 & 25 & 3 & 32 & 17 & $66^{*}$ & 15 \\
\hline L-Glutamine & 903 & 36 & $108^{*}$ & 27 & $126^{*}$ & 29 & $81^{*}$ & 21 & $123^{*}$ & 33 & $267^{*}$ & 55 \\
\hline L-Glutamate & 267 & 16 & $557^{\star}$ & 76 & $581^{*}$ & 77 & $387^{\star}$ & 41 & $417^{*}$ & 51 & $493^{*}$ & 86 \\
\hline Methionine: cystine & 0.23 & $0 \cdot 11$ & 0.57 & $0 \cdot 18$ & $1 \cdot 16^{*}$ & 0.17 & 0.21 & $0 \cdot 12$ & $1.01^{*}$ & 0.25 & $0.98^{*}$ & 0.17 \\
\hline Glutamine: cystine & 21.33 & $1 \cdot 80$ & $3.04^{*}$ & 0.98 & $3 \cdot 94^{\star}$ & 1.04 & $2 \cdot 40^{\star}$ & 0.58 & $4 \cdot 90^{\star}$ & 1.80 & $4 \cdot 47^{\star}$ & 1.14 \\
\hline
\end{tabular}

ND, not detected.

Mean values were significantly different from those of the control group: ${ }^{\star} P<0.05$

$\dagger$ Peripheral parenteral nutrition. For details of composition, see p. 174.

studied, L-glutamine concentration was significantly lower and L-glutamate concentration was significantly higher than in controls. It is important to emphasize that in normal subjects the L-glutamine concentration was similar to the values found in previous studies, but the values found in patients with abdominal inflammatory processes were very low.

\section{Whole-blood L-methionine : L-cystine and L-glutamine : $L$ - cystine ratios in patients}

The L-methionine: L-cystine ratio was 0.23 (SEM 0.11) in controls. The ratio in the appendicitis patients on admission was significantly higher than in controls. The ratio increased with time in the inflammatory patients, this being significantly higher $3 \mathrm{~d}$ after admission to hospital for all the cases studied. The L-glutamine: L-cystine ratio was 21.33 (SEM 1.80) in controls. However, this ratio was significantly lower on admission in all the patients studied when compared with controls (Table 2).

\section{Discussion}

$\gamma$-Cystathionase catalyses the conversion of L-cystathionine into L-cysteine, $\alpha$-ketobutyrate and $\mathrm{NH}_{4}{ }^{+}$, and its activity has been found in rat liver, whereas activity in rat kidney and heart is only $8 \%$ and $1 \%$ respectively of that found in rat liver (Matsuo \& Greenberg, 1958). In those situations where the liver $\gamma$-cystathionase activity has been shown to be inhibited, such as the neonatal period (Sturman et al. 1970; Zlotkin \& Anderson, 1982; Viña et al. 1995), surgical stress (Viña et al. 1992) and rats injected with propargylglycine (PPG) which is an inhibitor of $\gamma$ cystathionase (Triguero et al. 1997), the blood amino acid pattern is changed.

The clinical variables used to assess the nutritional status of patients are the serum albumin level and total lymphocyte count (Dickhaut et al. 1984; Klein et al. 1996). Values of albumin $>35 \mathrm{~g} / \mathrm{l}$, and the total lymphocytes count $>1500-2000$ cells $/ \mathrm{mm}^{3}$ reflect a good nutritional status. All our patients were well nourished and the
BMI was normal in all the cases studied, the only exception being a slight decrease in lymphocyte count and a moderate increase in the BMI in patients who had acute cholecystitis. Good nutritional status of the patients is important when studying amino acid and protein metabolism, because a recent 6 month randomized double-blind placebocontrolled trial found that protein supplementation $(20 \mathrm{~g} /$ d, 5 d per week) improved recovery from femoral fractures in an elderly population (Schürch et al. 1998).

Our patients had controlled parenteral nutrition for $3 \mathrm{~d}$ after their admission to hospital and the fact that the transsulfuration pathway is fully expressed in the liver makes this tissue an important factor in setting the blood concentration of $\mathrm{S}$-amino acids. The L-cystathionine concentration was significantly higher in pancreatitis or appendicitis than in controls, but no significant changes were found in cholecystitis when compared with controls. The L-serine concentration found in pancreatitis and cholecystitis was significantly higher when compared with controls, but no differences were found in appendicitis. However, the blood L-methionine: L-cystine ratio found in all patients studied was significantly higher than in controls.

In rats under surgical stress, the plasma L-cystine concentration was significantly lower than controls but in patients undergoing orthopaedic surgery or requiring resection of tumours, the plasma L-cystine was not significantly decreased when compared with controls (Crespo et al. 1997). Moreover, in rats injected with PPG which inhibits $\gamma$-cystathionase, the plasma L-cystine was unchanged when compared with controls. In all these cases, the L-methionine:L-cystine ratio was significantly higher than in controls (Triguero et al. 1997). As mentioned earlier, this concurs with our values (Table 2) in which the ratio was significantly higher in acute abdominal inflammatory stress when compared with controls. The ratio in the appendicitis patients was high from the time of admission to the hospital onwards due to the rapid evolution of their clinical situation. This emphasizes the importance of the evaluation of plasma L-methionine: Lcystine ratio instead of the free amino acid concentration 
when the study of the liver transsulfuration pathway is of interest.

In HIV infection, cancer, major injuries, sepsis, Crohn's disease, ulcerative colitis and in chronic fatigue syndrome, plasma L-cystine and L-glutamine concentrations are significantly lower and the L-glutamate concentration significantly higher than the values of control subjects. This is known as the 'low cystine and glutamine syndrome' (Drögue \& Holm, 1997; Hack et al. 1997). In young healthy subjects, during the post-absorptive period the muscle releases L-cysteine that is taken up by the liver. The L-cysteine catabolism in the liver generates an increased formation of sulfate and protons which down-regulates the rate of urea production because it decreases carbamoylphosphate synthetase 1 and therefore increases the Lglutamine synthesis. It is also concluded from the work of this group that the L-cysteine level is regulated primarily by the normal postabsortive skeletal muscle protein catabolism. In the patients mentioned earlier, it has been shown that plasma L-glutamine and L-cysteine and the glutamine: cysteine ratio are decreased and the L-glutamate concentration is elevated when compared with controls. The decreased blood L-glutamine:L-cystine ratio has been proposed as a good marker of an impaired hepatic Lcystine catabolism in the situations described earlier (Drögue \& Holm, 1997; Hack et al. 1997).

In our present study, the L-cystine concentration did not change but the L-glutamine concentration and the Lglutamine: L-cystine ratio decreased significantly and the L-glutamate concentration increased significantly in all the patients with acute abdominal inflammatory processes when compared with control subjects. This is another indication which shows that the $\mathrm{S}$-amino acid metabolism is modified in these patients but does not fully support the 'low cystine and glutamine syndrome' because L-cystine is not changed in our patients. This suggests that muscle is not affected and the liver is the organ responsible for these changes. In rats, the in vivo inhibition of the transsulfuration pathway with PPG which inactivates $\gamma$-cystathionase gives information related to the metabolic importance of this enzyme in the transsulfuration. At $4 \mathrm{~h}$ after the administration of PPG, liver $\gamma$-cystathionase activity was undetectable, L-cystathionine levels were significantly higher than in controls, L-cysteine was unchanged and liver GSH concentration was significantly lower than values found in control livers. The inhibition of $\gamma$ cystathionase was reflected in the blood levels of amino acids, because the L-methionine:L-cyst(e)ine ratio was significantly higher in the PPG-group than in the control group; blood concentration of L-cystathionine was also greater than control and L-cystine was unchanged. The administration of $\mathrm{N}$-acetylcysteine to PPG-treated rats reversed the changes in liver GSH (Triguero et al. 1997). The metabolic consequences of the inhibition of $\gamma$ cystathionase by PPG mimics in part the results found in our patients in the present study.

L-Cysteine availability is the rate-limiting step in GSH synthesis (Tateishi et al. 1974) and the restoration of GSH concentration in liver after inflammatory challenge is closely associated with an increased recycling and enhanced rate of synthesis when a high supply of substrates is available (Grimble, 1992; Hunter \& Grimble, 1997). In addition, it has been shown an increased requirement for Lcysteine during sepsis in rats (Malmezat et al. 1998), but in burn patients the rates of transmethylation, homocysteine remethylation and methionine oxidation were all substantially increased when compared with healthy adults ( $\mathrm{Yu}$ et al. 1993).

All the results found in our patients reported here show that plasma L-cystine concentration does not change when compared with controls, but the L-methionine: L-cystine ratio is increased. These results are similar to the values found in the rats injected with PPG, a powerful $\gamma$ cystathionase inhibitor. These results suggest the importance of using $N$-acetylcysteine as a required nutrient during those stressful situations in which the $\mathrm{S}$-amino acid metabolism is modified.

\section{Acknowledgements}

This work was supported by the Fondo de Investigación Sanitarias, Grants FIS-98/1461 and FIS 99/1157, Spain. Elisa Borrás was supported by a predoctoral fellowship from the Institute Danone, Spain.

\section{References}

Asagi K, Nakayama M, Kobayasi M, Awata S, Nakayama K \& Kodama H (1996) Content of sulfur amino acids and vitamin B6 and related enzyme activities in rats with chronic renal failure fed a high methionine diet. Nephron 74, 175-182.

Crespo ML, Giménez A, Bas T, García C, Puertes IR \& Viña JR (1997) Effect of nitrous oxide and propofol on amino acid metabolism in neoplasic patients. Nutrition and Cancer 27, 8083.

Dickhaut SC, DeLee JC \& Page CP (1984) Nutritional status: Importance in predicting wound healing in amputations. Journal of Bone and Joint Surgery (Am) 66, 71-75.

Drögue W \& Holm E (1997) Role of cystine and glutathione in HIV infection and other diseases associated with muscle wasting and immunological dysfunction. FASEB Journal 11, 1077-1089.

Erickson PF, Maxwell IH, Su LJ, Baumann M \& Glode LM (1990) Sequence of cDNA for rat cystathionase lyase and comparison of deduced amino acid sequence with related Escherichia coli enzymes. Biochemical Journal 269, 335-340.

Finkelstein JD (1990) Methionine metabolism in mammals. Journal of Nutritional Biochemistry 1, 228-237.

Grimble RF (1992) Dietary manipulation of the inflammatory response. Proceedings of the Nutrition Society 51, 285-294.

Hack V, Schmid D, Breitkreutz R, Stahlhenning C, Drings P, Kinscherf R, Taut F, Holm E \& Drögue W (1997) Cystine levels, cystine flux, and protein catabolism in cancer cachexia, HIV/SIV infection, and senescence. FASEB Journal 11, 84-92.

Hunter EAL \& Grimble RF (1997) Dietary sulfur amino acid adequacy influences glutathione synthesis and glutathionedependent enzymes during the inflammatory response to endotoxin and tumor necrosis factor-alpha in rats. Clinical Science 92, 297-305.

Klein JD, Hey LA, Yu ChS, Klein BB, Coufal FJ, Young EP, Marshall LF \& Garfin SR (1996) Perioperative nutrition and postoperative complications in patients undergoing spinal surgery. Spine 21, 2676-2682.

Kraus JP \& Rosenberg LE (1983) Cystathionine $\beta$-synthase from human liver: Improved purification scheme and additional 
characterization of the enzyme in crude and pure form. Archives of Biochemistry and Biophysics 222, 44-52.

Lu Y, O’Dowd B, Orrego H \& Israel Y (1992) Cloning and nucleotide sequence of human liver cDNA encoding for cystathionine lyase. Biochemical and Biophysical Research Communications 189, 749-758.

Malmezat T, Breuillé D, Pouyet C, Mirand PP \& Obled C (1998) Metabolism of cysteine is modified during the acute phase of sepsis in rats. Journal of Nutrition 128, 97-105.

Matsuo Y \& Greenberg DM (1958) A crystalline enzyme that cleaves homoserine and cystathionine. I. Isolation procedure and some physicochemical properties. Journal of Biological Chemistry 230, 545-560.

Nunn JF (1987) Clinical aspects of the interactions between nitrous oxide and vitamin B12. British Journal of Anaesthesiology 59, 3-13.

Rao AM, Drake MR \& Stipanuk MH (1990) Role of the transsulfuration pathway and of $\gamma$-cystathionase activity in the formation of cysteine and sulfate from methionine in rat hepatocytes. Journal of Nutrition 120, 837-845.

Schürch MA, Rizzoli R, Slosman D, Vadas L, Vergnaud P \& Bonjour JP (1998) Protein supplements increase serum insulinlike growth factor-I levels and attenuate proximal femur bone loss in patients with recent hip fracture. Annals of Internal Medicine 128, 801-809.

Storch KJ, Wagner DA, Burke JF \& Young V (1988) Quantitative study in vivo of methionine cycle in humans using [methyl-
2H3] and [1-13C]-L-methionine. American Journal of Physiology 18, E322-E331.

Sturman JA, Gaull G \& Raiha NCR (1970) Absence of cystathionase in human fetal liver: Is cystine essential? Science 169, 74-75.

Tateishi N, Higashi T, Shinya S, Naruse A \& Sakamoto Y (1974) Studies on the regulation of glutathione level in rat liver. Journal of Biochemistry (Tokyo) 75, 93-103.

Triguero A, Barber T, García C, Puertes IR, Sastre J \& Viña JR (1997) Liver intracellular L-cysteine concentration is maintained after inhibition of the transsulfuration pathway by propargylglycine in rats. British Journal of Nutrition 78, 823-831.

Viña J, Giménez A, Puertes IR, Gascó E \& Viña JR (1992) Impairment of cysteine synthesis from methionine in rats exposed to surgical stress. British Journal of Nutrition 68, 421429.

Viña J, Vento M, García-Sala F, Puertes IR, Gascó E, Sastre J, Asensi M \& Pallardó FV (1995) L-cystine and glutathione metabolism are impaired in premature infants due to cystathionase deficiency. American Journal of Clinical Nutrition 65, 1067-1069.

Yu YM, Burke JF \& Young VR (1993) A kinetic study of [L-2H3methyl] methionine in patients with severe burn injury. Journal of Trauma 35, 1-7.

Zlotkin SH \& Anderson GA (1982) The development of cystathionase activity during the first year of life. Pediatric Research 16, 165-168. 